\title{
Operative techniques of percutaneous endoscopic mini-hemilaminectomy using a uniportal approach in dogs
}

\author{
Hee-Sup MOON ${ }^{1}$, , Yong-Hyun HWANG ${ }^{1}$, Hee-Chun LEE ${ }^{1)}$ and Jae-Hoon LEE ${ }^{1) *}$ \\ ${ }^{1)}$ Institute of Animal Medicine, College of Veterinary Medicine, Gyeongsang National University, Jinju-si, 52828,
} South Korea

J. Vet. Med. Sci.

79(9): 1532-1539, 2017

doi: 10.1292/jvms.17-0148

Received: 27 March 2017

Accepted: 19 July 2017

Published online in J-STAGE: 31 July 2017
ABSTRACT. The present study aimed to investigate the technical feasibility of percutaneous endoscopic mini-hemilaminectomy via a uniportal approach, and to evaluate the possibility of decompression and endoscopic examination of the thoracic and lumbar spinal canals in small dogs during such procedures. Fresh canine cadavers of mixed-breed dogs $(n=7)$ were used in this study. Following injection of a barium and agarose mixture (BA-gel) to stimulate intervertebral disc herniation, percutaneous endoscopic mini-hemilaminectomy was performed using a lateral approach to the thoracic and lumbar vertebrae. BA-gel was removed to decompress the spinal cord using an elevator and rongeurs after mini-hemilaminectomy. Pre and post-operative computed tomography (CT) scans were obtained to evaluate surgical outcomes. Intra-operative complications, incision length, and procedure time were recorded. All procedures were completed with clear visualization of the spinal cord and floor of the spinal canal. The mean total operating time was $58.00 \pm 18.06 \mathrm{~min}$. Lengths of incision were under $1 \mathrm{~cm}$ in all dogs. Intra-operative complications included iatrogenic nerve root injuries caused by the micro-rongeur in two dogs. $\mathrm{CT}$ imaging revealed that removal of BA-gel resulted in sufficient spinal cord decompression. Our findings indicated that percutaneous endoscopic thoracolumbar mini-hemilaminectomy is feasible for spinal cord decompression and allows for adequate observation of the spinal canal. Thus, this technique may be an alternative surgical option for treatment of thoracolumbar disk disease in dogs.

KEY WORDS: dog, endoscopic mini-hemilaminectomy, minimally invasive surgery, neurosurgery

In human medicine, minimally invasive spinal procedures have gained popularity since the development of microendoscopic discectomy by Foley and Smith in 1999 [10]. Suggested advantages of endoscopic procedures over open surgery include less tissue trauma, minimal blood loss, improved visibility, easier access in obese patients, easier revision surgery owing to lower amounts of scar tissue, lower complication rates, ability to use local anesthesia, less pain, lower costs owing to shorter operating time and shorter inpatient stay [2].

Recently, minimally-invasive surgical procedures have also been emphasized for the reduction of postoperative morbidity and mortality in veterinary medicine $[15,23]$. While some studies have reported the success of endoscopic-assisted surgeries involving thoracoscopy, celioscopy and arthroscopy [1], few neurosurgical cases have been reported [5, 6, 12, 14, 22]. To the authors' knowledge, only five studies regarding endoscopic spine surgery have been published. Carrozo et al. performed endoscope-assisted thoracolumbar lateral corpectomy, exposing the surgical area using a Caspar cervical retractor [5], while Lockwood et al [14]. reported the success of endoscopic foraminotomy (EF) using a system of tubular dilators. In these previous studies [5, 6, 14, 22], an endoscope was used as a visual aid during surgery, while other instruments were required to perform the surgical procedures (e.g. Caspar cervical retractor, beveled tubular retractor). The percutaneous uniportal endoscopic procedure performed in the present study utilized a different approach than that typically encountered in veterinary medicine: All surgical procedures were performed through the port of the endoscope. More recently, Hwang et al. [12] utilized the same approach to perform percutaneous endoscopic thoracolumbar pediculectomy. Although the basic method for performing successful percutaneous endoscopic spinal surgery in live dogs was described, Hwang et al. [12] revealed several issues: (1) The surgical site did not correspond to the site of disc herniation, and (2) removal of herniated disc material was not demonstrated, as the procedure was performed in healthy, living dogs.

*Correspondence to: Lee, J.-H.: jh1000@gnu.ac.kr

(c2017 The Japanese Society of Veterinary Science

This is an open-access article distributed under the terms of the Creative Commons Attribution Non-Commercial No Derivatives (by-nc-nd) License. (CC-BY-NC-ND 4.0: https://creativecommons.org/licenses/by-nc-nd/4.0/) 
The purpose of the present study was to evaluate the feasibility of percutaneous endoscopic thoracolumbar minihemilaminectomy via a uniportal approach in small dogs. To overcome issues reported in other studies, we also aimed to demonstrate that an adequate window for the extraction of herniated disc material can be made by removing the injected barium and agarose mixture (BA-gel), thus resulting in successful decompression.

\section{MATERIALS AND METHODS}

\section{Experimental model of intervertebral disc disease (IVDD)}

Fresh canine cadavers were utilized for the present study. Mixed-breed dogs ( $\mathrm{n}=7)$ weighing $5.99 \pm 1.64 \mathrm{~kg}$ were euthanatized for reasons unrelated to the study and were determined to be free of spinal disease via CT scan. All dogs had normal vertebral structure, as determined via preoperative CT scan.

The experimental model of IVDD was created based on methods described by Lockwood et al. [14], with minor modifications. Agarose (0.15 g; USB Corporation, Cleveland, OH, U.S.A.) and powdered barium sulfate ( 0.4 g; Daejung, Korea) were mixed with tap water $(10 \mathrm{~m} l)$ in a $100-\mathrm{m} l$ flask, which was sealed with cotton to minimize vaporization. The mixture was heated to a boil using a microwave, inspirated into a $1-\mathrm{m} l$ syringe, and allowed to cool at room temperature.

Preliminary experiments were conducted using two canine cadavers to determine the proper volume of BA-gel, which was injected at five intervertebral sites using a $21 \mathrm{G}$ spinal needle. Each dog was positioned dorsoventrally, and the dorsal area around each injection site was clipped. The spinal needle was introduced through the interarcuate space, which allowed for access to the ventral portion of the spinal canal at each intervertebral space. Under fluoroscopic guidance, varying volumes of BA-gel were injected into five different epidural spaces (T9-10, T11-12, T13-L1, L2-3 and L4-5) to determine the appropriate volume of BA-gel. Following these preliminary investigations, BA-gel was injected at the T12-13 and L2-3 levels.

\section{Surgical technique}

Spinal cord compression was induced at two sites (T12-13, L2-3) in each dog in the same manner utilized to create the IVDD model. The side of BA-gel injection (right or left) was chosen randomly.

Operative instruments: The following instruments were obtained from Richard Wolf GmbH (Knittlingen, Germany). The discoscope utilized a rod lens with an outer diameter of $6.9 \mathrm{~mm}$, usable length of $207 \mathrm{~mm}$, and a vision angle of $25^{\circ}$. The device contained an intraendoscopic, eccentric working channel with a diameter of $4.1 \mathrm{~mm}$, as well as inlets for light and rinsing fluid. The working sleeve had an outer diameter of $8 \mathrm{~mm}$ and a beveled opening of $45^{\circ}$. A dilator (5.9 mm diameter, 2-channel), micro punch (290 mm length, $2.5 \mathrm{~mm}$ diameter), micro-rongeur (290 mm length, $2.5 \mathrm{~mm}$ diameter), and micro-bone punch (290 mm length, $2.5 \mathrm{~mm}$ diameter) were also used. Electrocautery (Trigger-Flex ${ }^{\circledR}$ Bipolar System, Elliquence, Netherlands) was performed to minimize hemorrhage and allow for a clear view through the endoscope. Burrs connected with a Combidrive ${ }^{\circledR}$ (Richard Wolf $\mathrm{GmBH}$, Knittlingen, Germany) were used for drilling. A passive irrigation system was also obtained from Richard Wolf GmBH.

Surgical approach under fluoroscopic guidance: The area around the surgical site was clipped, and each dog was positioned dorsoventrally. Two surgical procedures were performed in each dog (T12-13, L2-3). All procedures were performed by the same surgeon.

The spinal needle was placed dorsally under fluoroscopic guidance (KMC-650, COMED Medical Systems Co., Ltd., South Korea) for the identification of surgical sites. A $0.8-\mathrm{mm} \mathrm{K}$-wire was used to guide the position of the dilator. The K-wire was inserted at the superficial center of the foramen and advanced cranially or caudally to gain flexibility of the endoscope during the procedure. The tip of the K-wire was placed at any point between the caudal lamina of the cranial vertebra and the cranial lamina of caudal vertebra. Insertion was performed at angles of $0^{\circ}$ (for lumbar vertebra) and $20-30^{\circ}$ (for thoracic vertebra) to the parasagittal plane, as described by Hwang et al. [12]. Dorsoventral and lateral fluoroscopic images were obtained to confirm the surgical site. Once the K-wire had been positioned correctly, a skin incision $(<1 \mathrm{~cm})$ was made (Fig. 1A), following which the dilator and working sleeve were placed sequentially at the site of the K-wire under fluoroscopic guidance until bony resistance was encountered (Fig. 1B and 1C). The oblique side of the working sleeve was placed cranially to the cranial vertebra and caudally to the caudal vertebra to ensure adequate visualization of the surgical site through the endoscope. The K-wire and dilator were then removed, and the endoscope was passed through the working sleeve (Fig. 1D and 1E).

Percutaneous endoscopic mini-hemilaminectomy: Mini-hemilaminectomy was performed under continuous passive irrigation with normal saline. Prior to leveling epaxial muscle using the micro-rongeur and the micro-punch, electrocautery was performed to minimize hemorrhage and maintain adequate visualization of the surgical site (Fig. 2A). The burr connected to the Combidrive ${ }^{\circledR}$ was then advanced to the vertebral body. Pre-drilling was performed to account for potential loss of orientation using the screen. Drilling continued until the membrane of the inner cortex was exposed (Fig. 2B). The inner cortex membrane was cautiously broken using a trephine or micro-rongeur. The bony defect was extended cranially and centered caudally on the vertebral foramen, but did not include the zygapophyseal joint (articular process of the vertebra) (Fig. 2C). A micro-rongeur and micro-bone punch were used to create the bone window. An elevator (length: $290 \mathrm{~mm}$, diameter: $2.5 \mathrm{~mm}$ ) was used to remove the BA-gel, following which the spinal cord was elevated to ensure appropriate visualization of the ventral portion of the spinal canal in the event of residual BA-gel.

\section{Data collection}

Operative assessment: Operative complications, length of incision, and procedure time were recorded. Procedure time was 


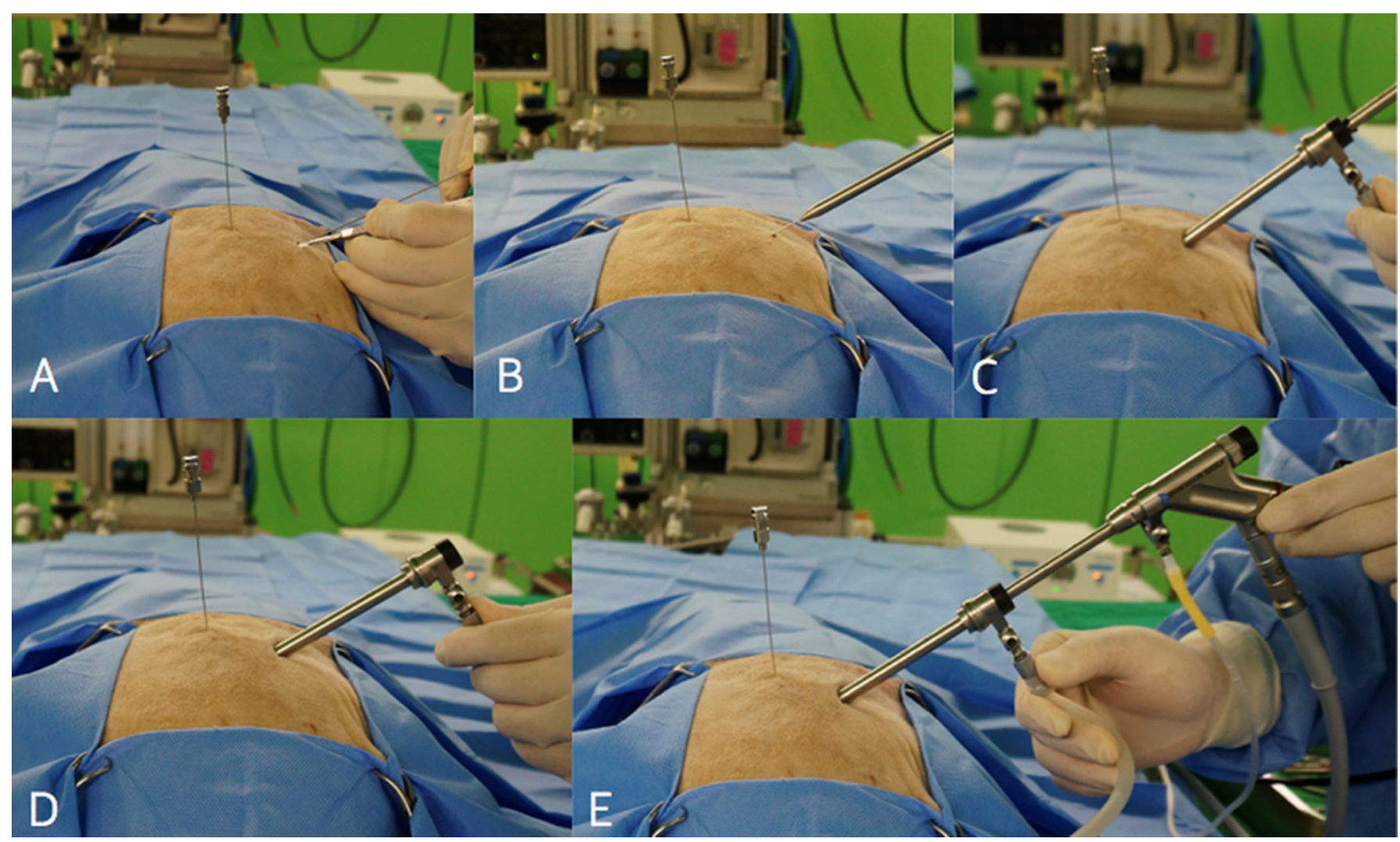

Fig. 1. Minimally-invasive approach to the lamina under fluoroscopic guidance. (A) Skin incisions of $1 \mathrm{~cm}$ were made following placement of a 0.8-mm K-wire on the lamina. (B) The dilator was advanced over the K-wire. (C) The working sleeve was placed over the dilator. (D) The K-wire and dilator were removed. (E) The endoscope was passed through the working sleeve.

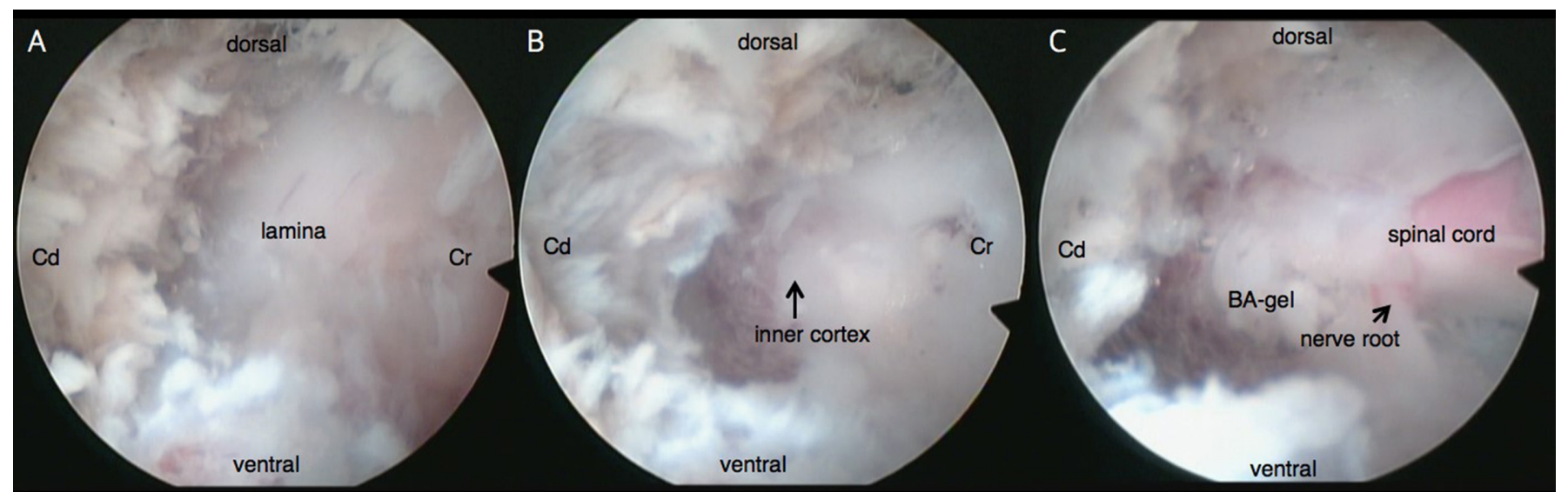

Fig. 2. View of thoracic spinal canal through the endoscope after percutaneous approach of the lamina at the level of T12-13. (A) The lamina was exposed after removal of epaxial muscle. (B) The outer cortex, cancellous bone, and inner cortex were sequentially removed during minihemilaminectomy. (C) Spinal cord, nerve root, and BA-gel were observed.

divided into four periods: endoscope approach time, bone exposure time, bone and BA-gel removal time and suture time. Surgical videos were also obtained using the endoscope. In addition, the feasibility of decompression by mini-hemilaminectomy and visualization of spinal nerve roots using an endoscope were evaluated.

CT scanning: Preoperative and postoperative CT images were obtained using a two-channel multi-detector row CT (MDCT) scanner (Somatom Emotion, Siemens Medical System, Erlangen, Germany) with the following parameters: $110 \mathrm{kV}, 36 \mathrm{mAs}$, and 1-mm or 2-mm slice thickness. CT scans of T11-12 were performed at the middle of the 10th thoracic vertebral body to the middle of the 13th thoracic vertebral body, while CT scans of L2-3 were performed at the middle of the 1st lumbar vertebral body to the 


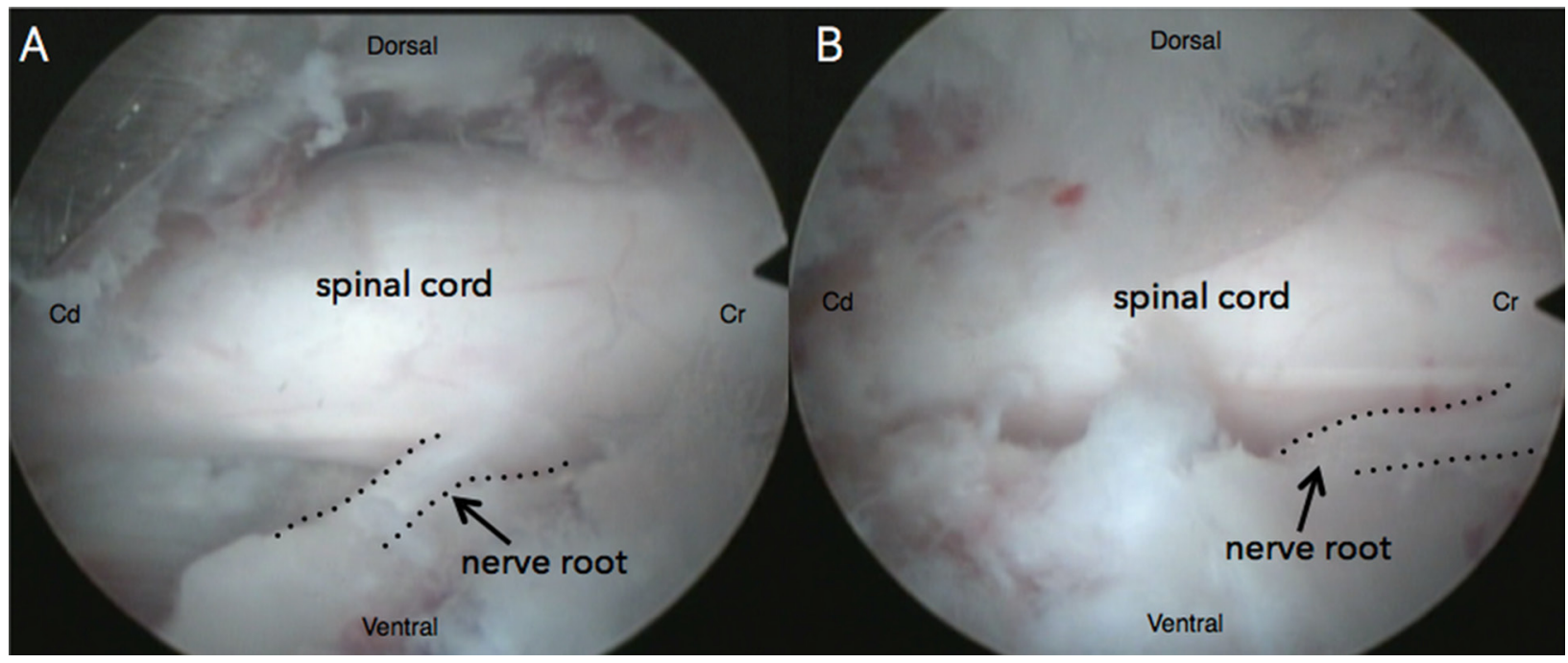

Fig. 3. View of thoracic (T12-13) and lumbar (L2-3) spinal canal after mini-hemilaminectomy using an endoscope. The spinal cord and nerve root (dotted line) were clearly identified. (A) Thoracic spinal canal. (B) Lumbar spinal canal.

Table 1. Procedure time and incision length for percutaneous endoscopic thoracolumbar mini-hemilaminectomy

\begin{tabular}{lcccccc}
\hline & \multicolumn{5}{c}{ Procedure time (min) } & \multicolumn{2}{c}{$\begin{array}{c}\text { Incision length } \\
(\mathrm{mm})\end{array}$} \\
\cline { 2 - 6 } & Endscope approach & Lamina exposure & $\begin{array}{c}\text { Removal of BA-gel } \\
\text { \& lamina }\end{array}$ & Suture & Total procedure & $8.29 \pm 0.49$ \\
Thoracic & $4.00 \pm 1.63$ & $6.57 \pm 2.07$ & $38.57 \pm 9.98$ & $1.00 \pm 00$ & $50.14 \pm 12.02$ & $8.29 \pm 0.39$ \\
Lumbar & $4.71 \pm 2.50$ & $9.71 \pm 5.77$ & $50.43 \pm 17.43$ & $1.00 \pm 00$ & $65.86 \pm 20.45$ & $8.29 \pm 0.43$ \\
All & $4.36 \pm 2.06$ & $8.14 \pm 4.47$ & $44.50 \pm 14.97$ & $1.00 \pm 00$ & $58.00 \pm 18.06$ &
\end{tabular}

Data are given as mean \pm SEM.

middle of the 3rd lumbar vertebral body. CT images were transferred to a separate workstation and analyzed with Lucion software (Infinitt Technlogy, Seoul, Korea).

Preoperative CT scans were performed following the injection of BA-gel to confirm normal vertebral structure and adequate spinal compression. The volume of injected BA-gel was also evaluated. Postoperative CT scans were performed to evaluate the amount of residual BA-gel, window size, and extent of decompression, which were calculated using Lucion software. The amount of BA-gel removed was calculated as the difference between the injected and residual volumes of BA-gel. Mann-Whitney analyzes were also conducted. Statistical analysis was performed using SPSS Version 23 (SPSS Inc., Chicago, IL, U.S.A.).

\section{RESULTS}

\section{Evaluation of IVDD modeling}

During the development of the IVDD model, an excessive volume of BA-gel was injected in the first trial, resulting in overlap throughout the spinal canal. Less than half of this volume was injected in the second trial. Consequently, we regarded $0.5 \mathrm{~m} l$ of BA-gel as an appropriate volume for compression of the spinal cord, which was confirmed via CT imaging. As it was impossible to prevent the spread of BA-gel from the intervertebral foramen to other intervertebral spaces, smaller amounts of BA-gel were injected onto the intervertebral space alone, although this did not result in spinal cord compression. Furthermore, residual amounts of BA-gel were observed owing to the spread of the gel across multiple intervertebral spaces.

\section{Surgical findings}

During the approach phase, the insertion of the longissimus tendon to the accessory process, vertebral foramen and lamina were observed through the endoscope. Adequate visualization of the spinal nerve root was achieved following creation of the bony defect (Fig. 3). The endoscope allowed for clear visualization and easy removal of the injected BA-gel in the ventral portion of the spinal canal, although the field of view was obstructed by friable BA-gel debris during removal of the gel. Visualization was easily recovered by increasing the velocity of lavage. Clear visualization was noted throughout the endoscopic procedure.

The length of the incision was less than $1 \mathrm{~cm}$ in each subject. The mean incision length was $8.29 \pm 0.43 \mathrm{~mm}$, which was slightly longer than the diameter of the working sleeve used in the present study (Table 1). The mean total operating times were $58.00 \pm$ $18.06 \mathrm{~min}$ and $50.14 \pm 12.02 \mathrm{~min}$ for thoracic vertebrae and $65.86 \pm 20.45 \mathrm{~min}$ for lumbar vertebrae. The endoscope approach 

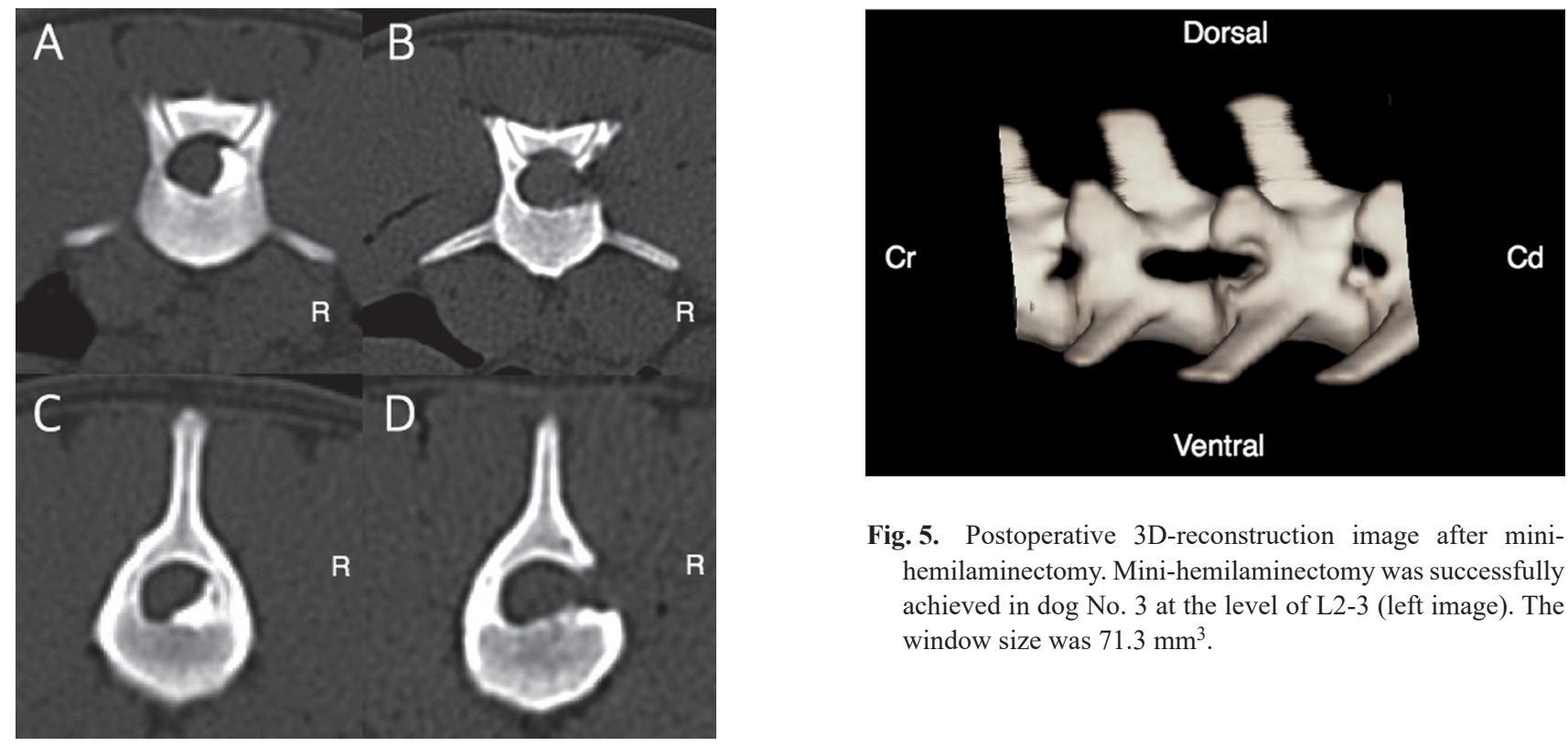

Fig. 5. Postoperative 3D-reconstruction image after minihemilaminectomy. Mini-hemilaminectomy was successfully achieved in dog No. 3 at the level of L2-3 (left image). The window size was $71.3 \mathrm{~mm}^{3}$.

Fig. 4. Preoperative and postoperative computed tomography (CT) images. (A) Preoperative thoracic images. (B) Postoperative thoracic images. (C) Preoperative lumbar images. (D) Postoperative lumbar images.

Table 2. The amounts of BA-gel and window size evaluated on computed tomography (CT) scan images

\begin{tabular}{lcccc}
\hline & $\begin{array}{c}\text { Pre-operative BA-gel } \\
\left(\mathrm{mm}^{3}\right)\end{array}$ & $\begin{array}{c}\text { Post-operative BA-gel } \\
\left(\mathrm{mm}^{3}\right)\end{array}$ & $\begin{array}{c}\text { Removed BA-gel } \\
\left(\mathrm{mm}^{3}\right)\end{array}$ & $\begin{array}{c}\text { Window size } \\
\left(\mathrm{mm}^{2}\right)\end{array}$ \\
\hline Thoracic & $265.71 \pm 48.26$ & $77.23 \pm 34.03$ & $187.09 \pm 41.95$ & $54.53 \pm 20.27$ \\
Lumbar & $295.71 \pm 83.04$ & $175.50 \pm 84.41$ & $120.20 \pm 33.74$ & $63.37 \pm 16.00$ \\
All & $280.71 \pm 67.08$ & $126.36 \pm 80.14$ & $153.64 \pm 50.42$ & $58.95 \pm 18.13$ \\
\hline
\end{tabular}

Data are given as mean \pm SEM.

time, bone exposure time, bone and BA-gel removal time and suture time for lumbar procedures were $4.71 \pm 2.50 \mathrm{~min}, 9.71 \pm 5.77$ $\min , 50.43 \pm 17.43 \mathrm{~min}$ and $1.00 \pm 0.00 \mathrm{~min}$, respectively. The endoscope approach time, bone exposure time, bone and BA-gel removal time and suture time for thoracic procedures were $4.00 \pm 1.63 \mathrm{~min}, 6.57 \pm 2.07 \mathrm{~min}, 38.57 \pm 9.98 \mathrm{~min}$ and $1.00 \pm 0.00$ $\mathrm{min}$, respectively. At both sites, bone and BA-gel removal accounted for the majority of procedure time, which increased when orientation was lost. As suturing required only a single, simple interrupted suture for all procedures, suture time was less than 1 min for both thoracic and lumbar sites (Table 1).

\section{Computed tomography (CT) scan findings}

There were significant differences between the volumes of injected and residual BA-gel for both thoracic $(P=0.001)$ and lumbar $(P=0.017)$ vertebrae. Removal of BA-gel was sufficient to achieve spinal cord decompression (Fig. 4). The average amount of BA-gel removed was $153.64 \pm 50.42 \mathrm{~mm}^{3}$ (Table 2). Residual BA-gel was observed for all procedures, though this did not appear to produce compression of the spinal cord. Postoperative lumbar CT illustrated that a significant residual volume of BA-gel in the area of the bony defect was detected in dog No 5. The average size of the vertebral window (bony defect) was $58.95 \pm 18.13 \mathrm{~mm}^{2}$ (Table 2). Three-dimensional reconstruction images were also obtained (Fig. 5). After decompression procedure by endoscope, residual amounts of BA-gel were observed owing to the spread of the gel across multiple intervertebral spaces, although no spinal cord compression was observed at the hemilaminectomy site.

\section{Complications}

Iatrogenic nerve root injuries caused by the micro-rongeur were noted in two cases involving thoracic procedures (Fig. 6). No nerve root injuries occurred during lumbar procedures. No other intraoperative complications were detected. Both complications occurred during removal of the muscle covering the bony defect with the micro-rongeur, which delayed removal of the BA-gel. The working sleeve had unintentionally shifted to an area near the opening of the nerve root, which was damaged during exposure of the vertebral lamina. 


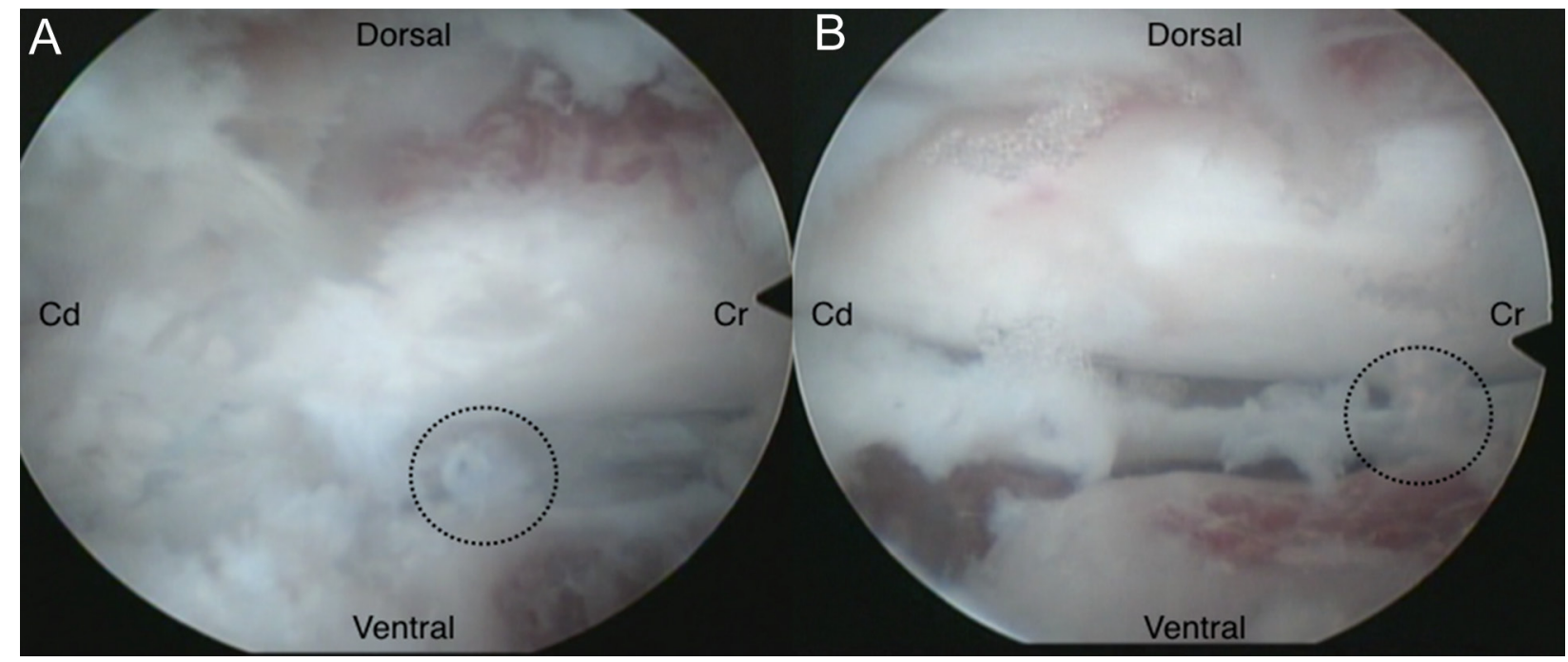

Fig. 6. Two iatrogenic nerve root injuries (dotted circle) caused by micro-rongeur in thoracic region. (A) The nerve root was injured during removal of the muscle covering the bony defect, which delayed removal of the BA-gel in dog No. 1. (B) The nerve root was injured during removal of the muscle following shifting of the working sleeve to the vertebral body in dog No. 5 .

\section{DISCUSSION}

In the present study, we aimed to highlight the feasibility of decompression via mini-hemilaminectomy by performing minimally invasive spine surgery at the thoracolumbar vertebrae of canine cadavers. Spinal compression was induced via injection of BA-gel, while decompression was achieved via removal of the gel using a micro-rongeur. Our findings suggest that sufficient decompression can be achieved via percutaneous endoscopic mini-hemilaminectomy.

Blind probing to remove herniated disc material increases the risk of venous sinus bleeding $[3,7,16]$. While standard operative techniques such as hemilaminectomy, dorsal laminectomy, and pediculectomy remove extruded disc material via blind probing, endoscopic procedures allow for adequate visualization of the ventral portion of the spinal cord. In the present study, we successfully removed disc material with the assistance of an endoscope, suggesting that endoscopic mini-hemilaminectomy is not only effective, but may also reduce the risk of venous sinus bleeding.

In the present study, we utilized an incision length of less than $1 \mathrm{~cm}$. In conventional hemilaminectomy, three cranial-to-caudal incisions are required [7]. According to Lockwood et al. [14], incision lengths were $2.4 \pm 0.04 \mathrm{~cm}$ in foraminotomy via an illuminated port (FP), $2.3 \pm 0.06 \mathrm{~cm}$ in EF and $5.3 \pm 0.31 \mathrm{~cm}$ in standard hemilaminectomy (SH) for dogs under $13 \mathrm{~kg}$. Although $\mathrm{EF}$ and $\mathrm{FP}$ required smaller incisions than $\mathrm{SH}$, the $\mathrm{EF}$ incision length of $2.3 \pm 0.06 \mathrm{~cm}$ made using a beveled tubular retractor was still markedly longer than that utilized in the present study [14]. Indeed, the incision length utilized in the present study was significantly shorter than those utilized in other types of minimally invasive surgery. Such differences in incision length may be due to differences in the approach systems used in each study: A more recent study by Hwang et al. [12] utilizing the same approach system as that of the present study reported similar results using an incision length of less than $1 \mathrm{~mm}$. The full-endoscopic approach system used in our study allows for the creation of incisions less than $1 \mathrm{~mm}$ in length, ensuring minimal invasiveness. Numerous advantages of minimally invasive techniques in human medicine may also be associated with such procedures in veterinary medicine, such as less tissue trauma, minimal blood loss, easier revision surgery owing to the presence of less scar tissue and less pain [2]. This is the main clinical advantage of our study, ultimately suggesting that such techniques can reduce morbidity in canine patients.

Large bone defects have also been associated with surgical failure [9]. According to a study by Huska et al. [11], a laminectomy membrane consisting of constrictive, fibrotic tissue results in surgical failure in human medicine, although only three cases have been reported in veterinary medicine [4]. This type of laminectomy membrane is thought to be caused by large bone defects [11]. Previous studies have utilized window sizes of $31.2 \mathrm{~mm}^{2}$ in $\mathrm{SH}, 22.8 \mathrm{~mm}^{2}$ in EF and $25.79 \mathrm{~mm}^{2}$ in percutaneous endoscopic pediculectomy $[12,14]$. However, we utilized a larger window size, as the amount of BA-gel removed in these previous studies was somewhat low (approximately $50 \mathrm{~mm}^{3}$ in SH and $75 \mathrm{~mm}^{3}$ in EF) [14]. Large defects have also been associated with spinal instability [21]. Studies have also indicated that large-breed dogs are more susceptible to spinal instability than small-breed dogs, and that hemilaminectomy extending up to three segments does not affect biomechanical stability in small-breed dogs [8, 19]. Factors affecting biomechanical stability include the state of zygapophyseal joints, intervertebral disc space, muscle tendons and ligaments [13]. Mini-hemilaminectomy preserves facet joints, and endoscopic techniques allow for minimal resection of muscle tendons and ligaments [5]. However, as small-breed dogs were used in the present study, spinal instability following percutaneous endoscopic mini-hemilaminectomy did not represent a major concern. These findings suggest that large bone defects allowed for greater removal of herniated disc material without influencing surgical success. 
Since 1934 [17], many studies of endoscopic spinal surgery have been reported in human medicine. Although various of methods and systems have been developed, common complications in humans include pyogenic infection of the disc space, dural tear, dysesthesia and thrombophlebitis [24]. The safe triangular zone must be approached to avoid nerve root injury in humans; [20] however, endoscopic instruments are too large to advance into this region in dogs. Few studies of endoscopic spinal surgery have been reported in veterinary medicine, and no complications other than fracture of the accessory process have been reported [12]. In the present study, we noted two instances of intraoperative complication, both of which involved iatrogenic nerve root injury in the thoracic region. The location of the vertebral canal relative to external landmarks at the vertebral junction is variable, largely due to the orientation of the ribs. Therefore, the surgeon's experience may have affected the complication rate in thoracic regions. To overcome such complications, surgeons should receive adequate training in endoscopic techniques, and adequate placement of the endoscope should be confirmed under fluoroscopic guidance prior to hemilaminectomy. After hemilaminectomy, the bone defect may be used to determine the orientation of the endoscope, which may be helpful in avoiding further complications.

In dogs with chronic intervertebral disc disease, herniated disc material tends to be located ventral to the midline and adhered to spinal cord [18]. Lateral corpectomy is recommended for the treatment of chronic disc disease, as it allows for easier access to the ventral portion of the spinal cord [18]. If additional surgery is required following percutaneous mini-hemilaminectomy, it is possible to extend the resection of the vertebral body ventrally. Thus, percutaneous endoscopic mini-hemilaminectomy can be converted to corpectomy for patients with adhesive disc herniation.

The present study possesses some limitations of note. First, we utilized dog cadavers, which did not allow us to assess postoperative complications or potential intraoperative complications such as bleeding. Furthermore, we did not perform MRI scanning or histopathologic examination after surgical procedures, which are essential for the assessment of postoperative spinal injury. In patients with multiple lesions, an open approach is preferred over an endoscopic procedure because each lesion demands a different approach, increasing operation times. The mean time to lamina exposure in the present study was 12.5 min. As endoscopic procedures may possess the advantages mentioned previously, percutaneous endoscopic mini-hemilaminectomy is recommended for patients with single lesions. However, access to lumbosacral and caudal cervical vertebrae using this approach is limited because of the presence of the sacrum and scapula. Further investigation is required to determine the most appropriate minimally invasive method for accessing lumbosacral and caudal cervical vertebrae. Although prophylactic fenestration is controversial, many surgeons perform fenestration following decompression. Although fenestration was not utilized in the present study owing to the lack of proper instruments for fenestration, we achieved adequate visualization of the intervertebral disc space. Lastly, we modified the experimental model of intervertebral disc herniation described by Lockwood et al. [14]. No model can truly equal an actual disc herniation. As in the previous report [14], the BA-gel spread along the canal beyond the boundaries of the disc space, and it was impossible to prevent the spread of BA-gel from the intervertebral foramen to other intervertebral spaces in the present study. In our study, although residual BA-gel did not appear to produce compression of the spinal cord on CT images at hemilaminectomy site, caution should be taken when applying the technique in clinical cases.

Our findings indicate that percutaneous endoscopic mini-hemilaminectomy via a uniportal approach was feasible in alleviating spinal compression with minimal invasiveness and the procedure at the lumbar are stable. However, further improvement needs to be made for the thoracic vertebrae to reduce iatrogenic injuries (e.g., never root injury). In conclusion, this technique made use of a small skin incision $(<1 \mathrm{~cm})$, was minimally invasive, required a relatively short surgical time (58 min), and allowed a good view during the operation. Furthermore, decompression was demonstrated by removing the simulated disc material through the port of the endoscope.

\section{REFERENCES}

1. Bernarde, A. and Viguier, E. 1999. A new limited transcutaneous endoscopically aided approach to the sacro-iliac joint: 6 cases. Vet. Surg. $28: 135$.

2. Birkenmaier, C., Chiu, J., Fontanella, A., Leu, H. and Ruetten, S. 2010. Guidelines for Percutaneous Endoscopic Spinal Surgery. ISMISS: 1-14.

3. Brisson, B. A. 2010. Intervertebral disc disease in dogs. Vet. Clin. North Am. Small Anim. Pract. 40: 829-858. [Medline] [CrossRef]

4. Brown, N. O., Helphrey, M. L. and Prata, R. G. 1977. Thoracolumbar disk disease in the dog: a retrospective analysis of 187 cases. Am. J. Vet. Res. 13: $665-672$.

5. Carozzo, C., Maitre, P., Genevois, J. P., Gabanou, P. A., Fau, D. and Viguier, E. 2011. Endoscope-assisted thoracolumbar lateral corpectomy. Vet. Surg. 40: 738-742. [Medline] [CrossRef]

6. Carozzo, C., Cachon, T., Genevois, J. P., Fau, D., Remy, D., Daniaux, L., Collard, F. and Viguier, E. 2008. Transiliac approach for exposure of lumbosacral intervertebral disk and foramen: technique description. Vet. Surg. 37: 27-31. [Medline] [CrossRef]

7. Coates, J. R. 2000. Intervertebral disk disease. Vet. Clin. North Am. Small Anim. Pract. 30: 77-110, vi. [Medline] [CrossRef]

8. Cudia, S. P. and Duval, J. M. 1997. Thoracolumbar intervertebral disk disease in large, nonchondrodystrophic dogs: a retrospective study. J. Am. Anim. Hosp. Assoc. 33: 456-460. [Medline] [CrossRef]

9. da Costa, R. C., Pippi, N. L., Graça, D. L., Fialho, S. A., Alves, A., Groff, A. C. and Rezler, U. 2006. The effects of free fat graft or cellulose membrane implants on laminectomy membrane formation in dogs. Vet. J. 171: 491-499. [Medline] [CrossRef]

10. Foley, K. T., Smith, M. M. and Rampersaud, Y. R. 1999. Microendoscopic approach to far-lateral lumbar disc herniation. Neurosurg. Focus 7: e5. [Medline] [CrossRef]

11. Huska, J. L., Gaitero, L., Brisson, B. A., Nykamp, S., Thomason, J. and Sears, W. C. 2014. Comparison of the access window created by hemilaminectomy and mini-hemilaminectomy in the thoracolumbar vertebral canal using computed tomography. Can. Vet. J. 55: 449-455. [Medline]

12. Hwang, Y. H., Lee, H. C. and Lee, J. H. 2016. Operative techniques and preliminary outcomes following percutaneous endoscopic thoracolumbar pediculectomy in dogs. Vet. Surg. 45 S1: O84-094. [Medline] [CrossRef] 
13. Kerwin, S. C., Levine, J. M. and Hicks, D. G. 2012. Thoracolumbar spine. pp. 449-475. In: Veterinary Surgery: Small Animal (Tobias, K. M. and Johnston, S. A. eds.), Elsevier, St, Louis.

14. Lockwood, A. A., Griffon, D. J., Gordon-Evans, W., Matheson, J. A., Barthélémy, N. and Schaeffer, D. J. 2014. Comparison of two minimally invasive approaches to the thoracolumbar spinal canal in dogs. Vet. Surg. 43: 209-221. [Medline] [CrossRef]

15. Mayhew, P. D. 2011. Complications of minimally invasive surgery in companion animals. Vet. Clin. North Am. Small Anim. Pract. 41: 1007-1021, vii-viii. [Medline] [CrossRef]

16. McCartney, W. 1997. Partial Pediculectomy for the Treatment of Thoracolumbar Disc Disease. VCOT Archive: 63-67.

17. Mixter, W. J. and Barr, J. S. 1934. Rupture of the intervertebral disc with involvement of the spinal cord. N. Engl. J. Med. 211: 210-214. [CrossRef]

18. Moissonnier, P., Meheust, P. and Carozzo, C. 2004. Thoracolumbar lateral corpectomy for treatment of chronic disk herniation: technique description and use in 15 dogs. Vet. Surg. 33: 620-628. [Medline] [CrossRef]

19. Olby, N., Levine, J., Harris, T., Muñana, K., Skeen, T. and Sharp, N. 2003. Long-term functional outcome of dogs with severe injuries of the thoracolumbar spinal cord: 87 cases (1996-2001). J. Am. Vet. Med. Assoc. 222: 762-769. [Medline] [CrossRef]

20. Perez-Cruet, M. J., Fessler, R. G. and Perin, N. I. 2002. Review: complications of minimally invasive spinal surgery. Neurosurgery 51 Suppl: S26S36. [Medline] [CrossRef]

21. Sharp, N. J. and Wheeler, S. J. 2005. Thoracolumbar disc disease. pp. 121-159. In: Small Animal Spinal Disorders: Diagnosis and Surgery. Elsvier Mosby, Philadelphia, Pennsylvania.

22. Wood, B. C., Lanz, O. I., Jones, J. C. and Shires, P. K. 2004. Endoscopic-assisted lumbosacral foraminotomy in the dog. Vet. Surg. 33: $221-231$. [Medline] [CrossRef]

23. Wu, X., Zhuang, S., Mao, Z. and Chen, H. 2006. Microendoscopic discectomy for lumbar disc herniation: surgical technique and outcome in 873 consecutive cases. Spine 31: 2689-2694. [Medline] [CrossRef]

24. Yeung, A. T. and Tsou, P. M. 2002. Posterolateral endoscopic excision for lumbar disc herniation: Surgical technique, outcome, and complications in 307 consecutive cases. Spine 27: 722-731. [Medline] [CrossRef] 